\title{
Viscosity, hard sphere diameter and interionic potential for liquid lead
}

\author{
G. Chaussemy \\ Laboratoire de Physique Electronique, Université Claude Bernard, \\ 43, boulevard du 11 novembre 1918, 69000 Villeurbanne, France
}

(Reçu le 13 février 1979, révisé le 14 mai 1979, accepté le 30 mai 1979)

\begin{abstract}
Résumé. - L'équation hybride de Macedo-Litovitz est utilisée conjointement avec un modèle sphères dures pour rendre compte de la viscosité du plomb entre $T=600$ et $1400 \mathrm{~K}$. Une compacité de 0,472 au point de fusion ainsi qu'une variation linéaire du diamètre de cœur dur $\sigma$ avec $T^{1 / 2}$ sont en très bon accord avec les résultats déduits de la théorie de Enskog et de la dynamique moléculaire. L'énergie d'activation : 0,07 eV trouvée est voisine de la hauteur de la barrière de potentiel interionique prévue à partir de l'équation de Percus-Yevick et des fonctions de corrélation déterminées par diffraction de neutrons.
\end{abstract}

\begin{abstract}
The Macedo-Litovitz equation for a hard sphere liquid provides a satisfactory model for the viscosity of lead up to $1400 \mathrm{~K}$. The packing fraction at the melting point $\left(\zeta_{\mathrm{m}}=0.472\right)$ and the $T^{1 / 2}$ temperature dependence of the hard core size $\sigma$ are in good agreement with other results deduced from Enskog's theory and molecular dynamics. The activation energy for viscosity $(0.07 \mathrm{eV})$ is similar to the height of the interionic potential barrier calculated from the correlation functions determined by means of neutron diffraction, and the Percus-Yevick theory.
\end{abstract}

1. Introduction. - Transport properties of high density liquids can be satisfactorily described in the framework of the hard sphere model by using a corrected Enshog's theory [1-3].

The hard core size $\sigma$ is close to intermolecular distances obtained from neutron diffraction measurements $[4,5]$. For liquid metals the temperature dependence of $\sigma$ is simply correlated with the interionic potential as shown by Protopapas et al. [6-8].

A more phenomenological approach to transport properties in liquids is the free volume formalism [9-12]. Macedo-Litovitz [13] and Chung [14] have reconsidered this formalism and proposed an hybrid equation for viscosity by using simple considerations concerning the interionic potential barrier. Both theories $[9,10$, $13,14]$ are of great interest to account for the viscosity of non associated or associated liquids, such as glass forming liquids $[15,16]$ and polymers $[13,17,18]$. In this paper the Arrhenius behaviour of kinematic viscosity for pure lead is well described up to $1400 \mathrm{~K}$ by a hard sphere model using both the MacedoLitovitz equation and a temperature dependent hard core size $\sigma$. The values of $\sigma$ and the height of the interionic potential barrier $\varepsilon^{*}$ are in good agreement with the results derived from neutron diffraction data [19].
2. Model. - 2.1 INTERATOMic POTENTIAL AND HARD CORE SIZE. - Figure 1 gives a schematic representation of interionic potential $\phi(r)$. The temperature dependence of $\sigma$ is well described by eq. (1) assuming that $\phi(r)$ is parabolic near $r_{0}$ and $k T$ is small [6] :

$$
\sigma=\sigma_{0}\left\{1-B\left(\frac{T}{T_{\mathrm{m}}}\right)^{1 / 2}\right\}
$$

$T_{\mathrm{m}}$ melting temperature.

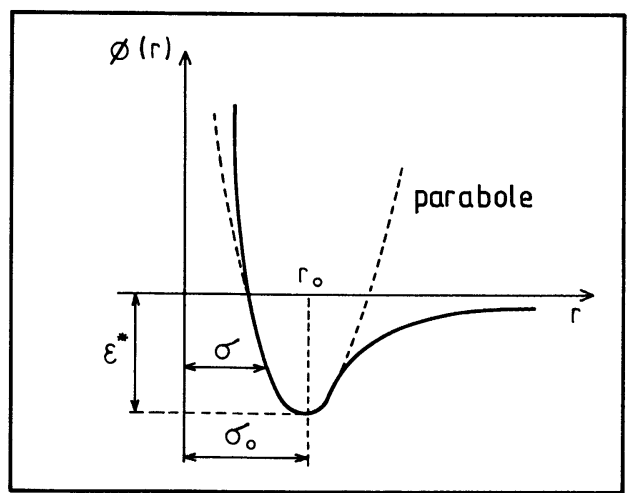

Fig. 1. - Schematic representation of the interionic potential $\phi(r)$ used in paragraph 2.1 . 
The hard sphere diameter $\sigma$ is the average distance of closest approach for repulsive collisions in the fluid [6]. If $p(E) \mathrm{d} E$ denotes the probability of finding an atom with energy in the interval $E, E+\mathrm{d} E$, we can write in the classical limit and in the case of a parabolic well [14] :

$$
p(E) \mathrm{d} E=\left(\frac{1}{2 k T}\right)\left(\frac{E}{k T}\right)^{2} \exp \left(-\frac{E}{k T}\right) \mathrm{d} E .
$$

2.2 Self-Diffusion COEFFICIENT. - If $p(v) \mathrm{d} v$ is the probability of finding a free volume between $v$ and $v+\mathrm{d} v[9]$ :

$$
p(v) \mathrm{d} v=\left(\frac{\gamma}{v_{\mathrm{F}}}\right) \exp \left\{-\frac{\gamma v}{v_{\mathrm{F}}}\right\} \mathrm{d} v
$$

$v_{\mathrm{F}}$ is the mean free volume and $\gamma$ a coefficient, arbitrarily taken equal to unity.

Diffusion in liquids in governed by two simultaneous events :

1) The presence of an adjacent free volume of size greater than $v^{*}$.

2) The acquisition of energy surpassing a critical value $\varepsilon^{*}$ by the diffusing atom.

The self-diffusion coefficient $D$ given by Turnbull in reference [15] can be rewritten in the following manner :

$$
D=\frac{1}{3} \frac{\gamma}{v_{\mathrm{F}}} \bar{u} \alpha \frac{1}{2 k T} \int_{v^{+}}^{\infty} \int_{\varepsilon^{*}}^{\infty} v p(E) \mathrm{d} E p(v) \mathrm{d} v
$$

assuming : (i) The displacement $a$ of an ion is a linear function $a=\alpha v$ of the free volume $v$, (ii) the ions move with the gas kinetic velocity $\bar{u}$.

By integrating eq. (4) gives :

$$
\begin{aligned}
D=\frac{1}{3} \gamma \bar{u} \alpha\left(v^{*}+\frac{v_{\mathrm{F}}}{\gamma}\right) & \left\{\frac{1}{2}\left(\frac{\varepsilon^{*}}{k T}\right)^{2}+\left(\frac{\varepsilon^{*}}{k T}\right)+1\right\} \times \\
& \times \exp -\left\{\frac{\gamma v^{*}}{v_{\mathrm{F}}}+\frac{\varepsilon^{*}}{k T}\right\} .
\end{aligned}
$$

Let $\zeta$ be the packing fraction of the hard sphere fluid. $\bar{v}_{\mathrm{m}}$ denotes the mean molecular volume and $\overline{v_{\mathrm{m}}}$ is the value of $\overline{v_{\mathrm{m}}}$ for $\zeta=\zeta_{0}=0.74$ (hard packing). Assuming $v_{\mathrm{F}}=\overline{v_{\mathrm{m}}}-\overline{v_{\mathrm{m}_{0}}}$ and $v^{*}=\overline{v_{\mathrm{m}_{0}}}$ eq. (5) is rewritten in the following form :

$$
\begin{aligned}
D=\frac{1}{18} \alpha \bar{u} \frac{\pi \sigma^{3}}{\zeta}\left\{\frac{1}{2}\left(\frac{\varepsilon^{*}}{k T}\right)^{2}+\left(\frac{\varepsilon^{*}}{k T}\right)+1\right\} \times \\
\times \exp -\left\{\frac{\zeta}{\zeta_{0}-\zeta}+\frac{\varepsilon^{*}}{k T}\right\} .
\end{aligned}
$$

2.3 VISCosITY. - For a hard sphere dilute gas [20] :

$$
D=1.019 \frac{3}{8 n} \frac{1}{\sigma^{2}}\left(\frac{k T}{\pi m}\right)^{1 / 2} .
$$

Eq. (7) is identical in the limit $\zeta \rightarrow 0$ and $\varepsilon^{*} \rightarrow 0$ to eq. (6). Then the value for $\alpha$ is : $0.4053 / \sigma^{2}$.

The assumption $a=\alpha v$ is strictly valid only for dense fluids (if $v \rightarrow 0$ ), consequently this estimation for $\alpha$ is only a rough approximation.

For spherical molecules, the Stokes-Einstein relationship is valid :

$$
\frac{\eta D \sigma}{k T}=C
$$

where $C$ is a constant. ( $C=1 / 2 \pi$ for liquid metals [7]) and $\eta$ is the shear viscosity coefficient.

The kinematic viscosity is expressed by :

$$
\begin{array}{r}
v=\frac{\eta}{d}=\frac{A T^{1 / 2}}{\left[1+\left(\frac{\varepsilon^{*}}{k T}\right)+\frac{1}{2}\left(\frac{\varepsilon^{*}}{k T}\right)^{2}\right]\left(\frac{\zeta}{d}\right)^{1 / 2} \times} \\
\times \exp \left[\frac{\zeta}{\zeta_{0}-\zeta}+\frac{\varepsilon^{*}}{k T}\right]
\end{array}
$$

$A=0.4065 \times 10^{-4}$ for lead, $d$ is the density.

3. Results and discussion. - 3.1 Results. - The kinematic viscosity $v$ of liquid lead was measured with a Meyer Schvidkovskii torsional oscillating cup viscometer. The absolute value of $v$ was determined with an estimated error of $10 \%$ but for the temperature dependence of the viscosity the relative precision is $2 \%$. In the range of measurements from melting point to $800^{\circ} \mathrm{C}$ our results are similar to those in references [3] and [7] (Fig. 2).

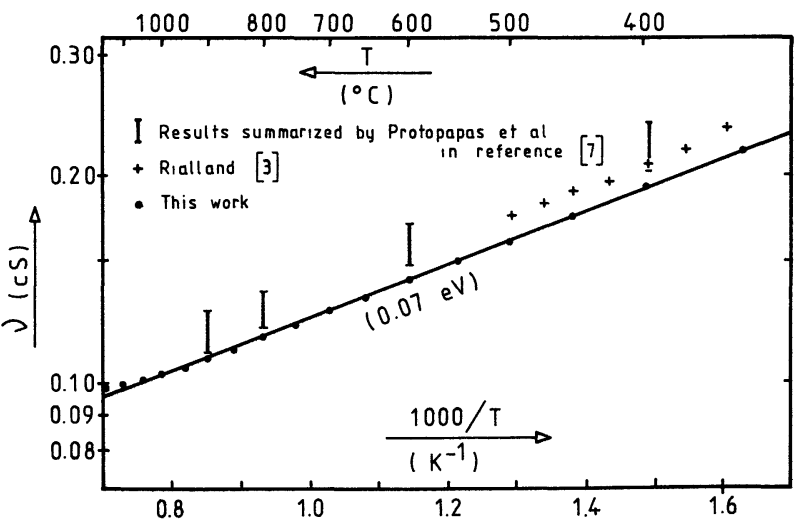

Fig. 2. - Experimental values of kinematic viscosity $v$ for liquid lead as a function of $1 / T$. The straight line corresponds to the Arrhenius law $v=v_{0} \exp (W / k T)$ with an apparent activation energy $W=0.07 \mathrm{eV}$.

In figure 2 we plot $\log v$ versus $1 / T$. Experimental $v$ values are also given in table $\mathrm{I}$. The apparent activation energy of an Arrhenius law $v=v_{0} \exp (W / k T)$ is $W=0.07 \mathrm{eV}$. $\zeta$ and $\sigma$ were calculated from experimental values of $v$ and eq. (9). $\varepsilon^{*}$ is an adjustable parameter. Taking $\varepsilon^{*}=0.06 \mathrm{eV}$ and $\zeta=0.465$ at 
Table I. $-v_{\exp }=$ experimental values of kinematic viscosity of figure $1 ; v_{\text {calc }}=$ kinematic viscosity calculated with eq. (9); $\sigma_{\text {calc }}=$ hard sphere diameter deduced from eq. (1); $\zeta=$ packing fraction used in eq. (9).

\begin{tabular}{|c|c|c|c|c|c|}
\hline$T\left({ }^{\circ} \mathrm{C}\right)$ & $v_{\text {exp }}=v_{\text {calc }}(\mathrm{cS})$ & $\zeta$ & $\sigma(\AA)$ & $\sigma_{\text {calc }}(\AA)$ & $\frac{\sigma-\sigma_{\text {calc }}}{\sigma} \times 10^{3}$ \\
\hline- & - & - & - & - & - \\
\hline 327 & & $0.472\left(^{*}\right)$ & & $3.072\left(^{*}\right)$ & \\
\hline 340 & 0.215 & 0.465 & 3.061 & 3.065 & -1.4 \\
\hline 400 & 0.192 & 0.451 & 3.037 & 3.036 & +0.5 \\
\hline 450 & 0.174 & 0.438 & 3.014 & 3.012 & +0.7 \\
\hline 500 & 0.160 & 0.425 & 2.990 & 2.989 & +0.3 \\
\hline 550 & 0.150 & 0.414 & 2.970 & 2.967 & +1.1 \\
\hline 600 & 0.141 & 0.403 & 2.950 & 2.946 & +1.5 \\
\hline 650 & 0.132 & 0.390 & 2.924 & 2.925 & -0.2 \\
\hline 700 & 0.127 & 0.381 & 2.908 & 2.905 & +1.1 \\
\hline 750 & 0.121 & 0.370 & 2.886 & 2.885 & +0.3 \\
\hline 800 & 0.116 & 0.360 & 2.866 & 2.866 & 0.0 \\
\hline 850 & 0.111 & 0.348 & 2.840 & 2.847 & -2.5 \\
\hline 900 & 0.108 & 0.339 & 2.821 & $2: 828$ & -2.6 \\
\hline 950 & 0.105 & 0.331 & 2.805 & 2.810 & -1.9 \\
\hline 1000 & 0.103 & 0.323 & 2.788 & 2.793 & -1.5 \\
\hline 1050 & 0.101 & 0.316 & 2.774 & 2.775 & -0.4 \\
\hline 1100 & 0.100 & 0.310 & 2.763 & 2.758 & +1.6 \\
\hline 1150 & 0.099 & 0.304 & 2.751 & 2.742 & +3.5 \\
\hline
\end{tabular}

$\left(^{*}\right)$ The packing fraction and the hard core size at the melting point are extrapolated.

$340{ }^{\circ} \mathrm{C}$ the temperature dependence of $\sigma$ agrees well with eq. (1) written in the following form :

$$
\sigma_{\text {calc }}=3.68\left(1-0.166\left(\frac{T}{600}\right)^{1 / 2}\right) \quad T_{\mathrm{m}}=600 \mathrm{~K} \text {. }
$$

As shown in table $\mathrm{I},\left(\sigma-\sigma_{\text {calc }}\right) / \sigma$ does not exceed $0.25 \%$ up to $1100{ }^{\circ} \mathrm{C}$. The variation of $\sigma$ in the range of measurements is approximately $10 \%$, then the subsequent precision in the agreement with eq. (1) is about $2.5 \%$.

3.2 Discussion. - Previously, using total and direct correlation functions obtained from neutron diffraction experiments North et al. [19] have calculated a Percus-Yevick interionic pair-potential. Assuming $\sigma_{0}$ is the position of the potential well and $\varepsilon^{*}$ the height of the potential barrier our results are in very good agreement with [19].

By comparing $\sigma$ with $r_{\max }$, the first peak position of radial distribution function [19] as shown in

Table II. $-\sigma=$ hard core diameter of table I; $r_{\max }=$ position of the first peak of the radial distribution function given by North et al. [19].

$\begin{array}{rcc}T\left({ }^{\circ} \mathrm{C}\right) & r_{\max }(\AA) & \sigma / r_{\max } \\ - & - & 0.90 \\ 340 & 3.40 & 0.89 \\ 600 & 3.32 & 0.87 \\ 780 & 3.30 & 0.86\end{array}$

table II we found 0.9 for $\sigma / r_{\max }$ at $340^{\circ} \mathrm{C}$. This value has been observed for other liquids and salts at the melting point [5], and considered as an indication of a relation between $\sigma$ and mean spacing of shortrange order.

The temperature dependence of $\sigma$ is consistent with the hypothesis of paragraph 2.1 and reference [6].

The packing fraction $\zeta_{\mathrm{m}}=0.472$ at the melting temperature corresponds to the usual assumptions for liquid metals $[6,7,21]$ and fits very well Lindemann's law [22, 23].

For $B$ in eq. (1) Protopapas et al. [6] predicted a value equal to 0.112 for all metals, we found 0.166 . The difference is about $50 \%$ but only $6 \%$ for $\sigma_{0}$.

The precision $\Delta \zeta / \zeta$ on the calculated packing fraction which results from the experimental error $\Delta v / v=10 \%$ mentioned above is $2 \%$ at $340{ }^{\circ} \mathrm{C}$ and $6 \%$ at $1100{ }^{\circ} \mathrm{C}$, but the corresponding precision $\Delta \sigma / \sigma$ is only $1 / 3 \Delta \zeta / \zeta$. For a given value of $\varepsilon^{*}$ the effects of $\Delta v / v$ on the preceding discussion are small : viscosity is very sensitive to variations of the hard core size. However, at $340{ }^{\circ} \mathrm{C}$ for the value $\zeta=0.465$, the relative variation $\Delta \varepsilon^{*} / \varepsilon^{*}$ giving rise to $\Delta v / v=10 \%$ is about $30 \%$ : viscosity is less sensitive to variation of $\varepsilon^{*}$, if $\varepsilon^{*}$ is small.

4. Conclusion. - The model gives a simple connection between viscosity and interionic pair potential. Both for non associated and associated liquids, viscosity measurements provide a practical method for investigating interatomic forces. For lead the agree- 
ment with other observations is quite satisfactory and the $\varepsilon^{*}$ and $\zeta_{\mathrm{m}}$ values present some physical interest. However the choice of $\varepsilon^{*}$ and $\zeta_{\mathrm{m}}$ acting as adjustable parameters for viscosity, is not sufficiently critical and must be completed with data given by other experiments such as neutron diffraction.

\section{References}

[1] Dymond, J. H., J. Chem. Soc. Faraday Trans. II 68 (1972) 1789.

[2] Dymond, J. H., Chem. Phys. 60 (1974) 969.

[3] Rialland, J. R., Thesis, Orsay (1977).

[4] Van Loef, J. J., Da Silva, M. J. and Tricht, J. B., Chem. Phys. Lett. 28 (1974) 508.

[5] VAN LOEF, J. J., Z. Naturforsch. 31a (1976) 967.

[6] Protopapas, P., Andersen, H. C. and Parlee, N. A. D., J. Chem. Phys. 59 (1973) 15.

[7] Protopapas, P., Andersen, H. C. and Parlee, N. A. D., Chem. Phys. 8 (1975) 17.

[8] Protopapas, P. and Parlee, N. A. D., Chem. Phys. 11 (1975) 201.

[9] Cohen, M. H. and Turnbull, D., J. Chem. Phys. 31 (1959) 1164.

[10] Turnbull, D. and Cohen, M. H., J. Chem. Phys. 34 (1961) 120.

[11] Naghizadeh, J., J. Appl. Phys. 35 (1964) 1162.

[12] Matheson, A. J., J. Chem. Phys. 44 (1966) 695.

[13] Macedo, P. B. and Litovitz, T. A., J. Chem. Phys. 42 (1965) 245.

[14] Chung, H. S., J. Chem. Phys. 44 (1966) 1362.

[15] Turnbull, D. and Cohen, M. H., J. Chem. Phys. 52 (1970) 3038.

[16] Laugier, A., Chaussemy, G. and Fornazero, J., J. NonCryst. Solids 23 (1977) 419.

[17] Sanchez, I. C., J. Appl. Phys. 45 (1974) 4204.

[18] Rialland, J. F. and Perron, J. C., Proceedings of the 6th Int. Conf. on Amorph and Liq. Semicond., Ed. B. A. Kolomiets Leningrad, 1976.

[19] North, D. M., Enderby, J. E. and EgelstafF, P. A., J. Phys. C (Proc. Phys. Soc.) 1 (1968) 1075.

[20] Chapman, S. and Cowling, T. G., The mathematical theory of non-uniform gases (Cambridge at the University Press) 1970.

[21] FABER, T. E., Introduction to the theory of liquid metals (Cambridge at the University Press) 1972.

[22] Ross, M., Phys. Rev. 184 (1969) 233.

[23] Van Loef, J. J., J. Chem. Phys. 61 (1974) 1605. 